\title{
Corrosion Inhibition of Mild Steel by Poly(butylene succinate)-L-histidine Extended with 1,6-diisocynatohexane Polymer Composite in $1 \mathrm{M} \mathrm{HCl}$
}

\author{
M. G. Tsoeunyane $\mathbb{D}^{1},{ }^{1}$ M. E. Makhatha, ${ }^{1}$ and O. A. Arotiba ${ }^{2,3}$ \\ ${ }^{1}$ Department of Metallurgy, University of Johannesburg, South Africa \\ ${ }^{2}$ Department of Applied Chemistry, University of Johannesburg, South Africa \\ ${ }^{3}$ Centre for Nanomaterials Science Research, University of Johannesburg, South Africa \\ Correspondence should be addressed to M. G. Tsoeunyane; m.tsoeunyane.mg@gmail.com
}

Received 23 October 2018; Revised 23 January 2019; Accepted 4 February 2019; Published 3 March 2019

Academic Editor: Michael J. Schütze

Copyright ( 2019 M. G. Tsoeunyane et al. This is an open access article distributed under the Creative Commons Attribution License, which permits unrestricted use, distribution, and reproduction in any medium, provided the original work is properly cited.

\begin{abstract}
The ecofriendly poly(butylene succinate) extended with 1,6-diisocynatohexane composted with L-histidine (PBSLH) polymer composite was synthesized by condensation polymerization. The polymer composite was characterized by Fourier transform infrared (FTIR), X-ray diffraction (XRD), and scanning electron microscope (SEM-EDX). The inhibition action of the polymer composite was investigated by conventional weight loss, potentiodynamic polarization, variable amplitude micro (VASP), and electrochemical impedance spectroscopy (EIS). The maximum corrosion inhibition efficiency of $78 \%$ was obtained at concentration level of $600 \mathrm{ppm}$. The results revealed PBSLH as a mixed type corrosion inhibitor. The thermodynamic and kinetic parameters also revealed adsorption of PBSLH on to mild surface as exothermic and the adsorption was conformed to Langmuir model. The morphology of mild steel coupons was investigated by SEM and atomic force microscope (AFM) and the results showed PBSLH to have inhibited corrosion on mild steel in $1 \mathrm{M}$ HCL.
\end{abstract}

\section{Introduction}

The use of corrosion inhibitors to protect metals and their alloys against corrosion had been accounted to be the best practical method [1]. A corrosion inhibitor is depicted as the substance that when added in small concentrations to a corrosive media reduces the corrosion rate of the metals $[2,3]$. Several corrosion inhibitors are organic compounds in nature [4]; it is generally assumed that corrosion inhibition is performed by adsorption of molecules on the metal-solution interface $[5,6]$. The efficiency of corrosion inhibitors depends on their molecular structure.

It had been reported by [7-9] that organic corrosion inhibitors by and large have heterogeneous atoms being $\mathrm{O}$, $\mathrm{N}, \mathrm{S}$ and $\mathrm{P}$ these atoms are found to have high basicity and electron density which aid in the corrosion inhibition of metals and/or alloys. Likewise compounds with the conjugated pi bond system had been reported to have the ability to inhibit corrosion. It has likewise been expressed that there are factors that govern the corrosion inhibition. These factors include chain length, the size of the molecule, bonding/conjugate system with the molecule, cross-linking ability, and solubility in the environment.

Corrosion merits researching in light of the fact that corrosion issues present an extensive portion of the absolute expenses in various assets and production companies every year worldwide [6]. This is a problem for almost every industry dealing with metallic structures ranging from transportation, oil, and gas to structure building industries [10]. Corrosion urges spontaneous shut down of plants and causes awful incidents that ultimately result in environmental damage and human causalities [11]. It has been accounted for that $25 \%$ of fatalities experienced in gas and oil industries are mostly because of corrosion.

Through the decades, distinctive techniques for corrosion reduction or prevention had been reported by many scientists and engineers. This incorporates however not just cathodic protection, anodic protection, utilization of 
coatings, environmental treatment, and corrosion inhibitors $[12,13]$. The majority of the corrosion prevention methods have their own downsides; however among all protection methods, the use of corrosion inhibitors had proven to be the most effective method $[1,14,15]$ for most part of the internal corrosion of materials in acidic medium [16]. This suggests that the use of corrosion inhibitors to prevent/reduce corrosion rate is of extraordinary significance explicitly in pipeline industries.

As the commitment to new improvement of green corrosion inhibitors, we in this report showcase the synthesis of polybutylene succinate extended with 1,6-diisocynatohexane -L-histidine composite (PBSLH). The newly synthesized composite was characterized by FTIR, XRD, and SEM/EDX. The inhibition effect of PBSLH was investigated by gravimetric analysis and electrochemical analysis in $1 \mathrm{M} \mathrm{HCl}$ with different concentration of PBSLH as the corrosion inhibitor. The possible mechanism of PBSLH adsorption on to mild steel surface was also explored. The average roughness of the mild steel specimen was characterized by both SEM and AFM.

\section{Experiments}

2.1. Synthesis of PBSLH. In all the experiments, distilled water was utilized to prepare the solutions. The corrosion inhibitor was synthesized from poly(butylene-succinate) extended with 1,6-diisocynatohexane (PBS) and L-histidine. The paste of PBS was made in chloroform; then $5 \%$ solution of PBS in $1 \mathrm{M}$ oxalic acid was mixed with $1 \%$ sodium lauryl to increase its solubility. To the mixture $1 \%$ L-histidine was added and the mixture was stirred for 2 hours. The temperature of the mixture was maintained between 0 and $5^{\circ} \mathrm{C}$. Freshly prepared ammonium-peroxodisulphate equimolar to L-histidine was added dropwisely to the cold mixture with constant stirring. The mixture was stirred for 2 hours and maintained at a $\mathrm{pH}$ range of 8 to 9 . The mixture was refrigerated for the day and the polymer composite was obtained by precipitation with a nonsolvent liquid, filtered, washed, tried and stored. The newly synthesized PBSLH composite was subjected to characterization by FTIR, XRD, and SEM-EDX analysis to confirm polymerization.

2.2. Electrode and Electrolytes. The test material was mild steel having the chemical composition of $\mathrm{Mn}-0.249, \mathrm{C}-$ $0.061, \mathrm{P}-0.015, \mathrm{Cr}-0.027, \mathrm{~S}-<0.1, \mathrm{Ni}-0.016, \mathrm{Si}-0.013$, Mo - 0.024, Al - 0.040, Co - 0.011, Mg - 0.013, Se - 0.039, and $\mathrm{Zn}-0.012$ with the balance as $\mathrm{Fe}$ (wt.\%). The dimensions of mild steel coupons were $2.4 \times 1.2 \times 0.2 \mathrm{~cm}$ for gravimetric and surface analysis while $12.574 \mathrm{~cm}^{2}$ was the area exposed for those selected for electrochemical analysis. Mild steel coupons were initially polished with emery paper $(600,800$, and 1200) starting with coarse until the mirror appearance was obtained. The coupons were washed with distilled water several times and degreased with acetone before allowing them to dry at room temperature. Analytical grade $37 \% \mathrm{HCl}$ was used to prepare $1 \mathrm{M} \mathrm{HCl}$ and the inhibitor concentration was varied from $200 \mathrm{ppm}$ to $1000 \mathrm{ppm}$.
2.3. Weight Loss Method. The rectangular strips were immersed in duplicates in $1 \mathrm{M} \mathrm{HCl}$ in the absence and presence of different concentrations of PBSLH. The concentration optimization of the inhibitor was done at 3 hours period. The effect of immersion time was performed between 3 and 24 hours; the experiments were carried out at room temperature. The water bath was used to stabilize the temperature. Prior immersion into the electrolyte, the coupons were stamped and weighed. The coupons were taken out after designated period; corrosion products were removed and reweighed. From the data obtained, parameters such as corrosion rate (CR) in mmpy, Inhibition efficiency (IE), and surface coverage $(\theta)$ were calculated by

$$
\begin{aligned}
C R & =\frac{87.6 \mathrm{~W}}{t A d} \\
\% I E & =\frac{C R_{0}-C R_{\text {in }}}{C R_{0}} \times 100 \\
\theta & =\frac{C R_{0}-C R_{\text {in }}}{C R_{0}}
\end{aligned}
$$

where $\mathrm{W}$ is the weight loss of mild steel in grams, $\mathrm{A}$ is the area, $d$ is the density, and $t$ is the total time of immersion while $\mathrm{CR}_{0}$ and $\mathrm{CR}_{\text {in }}$ are corrosion rates of mild steel in the absence and presence of the inhibitor. The same process was repeated for temperature studies with the immersion period of 0.5 hours, where the thermodynamics and kinetics of the inhibitor adsorption were explored.

2.4. Electrochemical Measurements. Bio-Logic single channel potentiostat SP-150 supported with EC-Lab software was utilized for data acquisition and analysis. The corrosion flat cell with three-electrode system consisting of platinum gauze was used as the counter electrode, a saturated calomel electrode (SCE) as the reference, and mild steel as the working electrode. Prior analysis, the steady state potential was obtained by letting the mild still to corrode freely in an open circuit potential (OCP) versus SCE. The temperature was maintained at $25^{\circ} \mathrm{C}$ in all test analysis. The polarization studies were carried out within the potential range of -250 $\mathrm{mV}$ to $+250 \mathrm{mV}$ (vs SCE) with respect to the steady state potential at the scan rate of $1 \mathrm{mVs}^{-1}$. Both anodic and cathodic Tafel plots were extrapolated to obtain parameters such as corrosion potential $\left(E_{\text {corr }}\right)$, corrosion current density $\left(I_{\text {corr }}\right)$, and Tafel slopes. The IE was calculated form the measured $I_{\text {corr }}$ values using the relationship,

$$
I E_{p}=\frac{I_{c o r r}-I_{c o r r}^{\prime}}{I_{c o r r}} \times 100
$$

where $I_{c o r r}$ and $I_{\text {corr }}^{\prime}$ are corrosion density without and with the presence on corrosion inhibitor, respectively.

To determine the total polarization resistance $\left(R_{p}\right)$ of mild steel dissolution, variable sinusoidal amplitude microPolarization (VASP) was the technique that was employed. Resistance polarization refers to the potential drop due to high resistivity of the electrolyte or the film formation on the surface of the electrode that increases the insulation on 
the electrode/solution interface. This implies that the higher $\mathrm{R}_{\mathrm{p}}$ in terms of corrosion of materials, the more material is protected. VASP fit that is found on EC-Lab software would be used to determine $R_{p}$.

The electrochemical impedance spectroscopy (EIS) measurements were performed at OCP in a frequency range from $100 \mathrm{KHz}$ to $0.1 \mathrm{~Hz}$ and the system was perturbed with sine wave of amplitude of $10 \mathrm{mV}$. The charge transfer resistance was obtained by fitting the semicircles of Nyquist representation used to calculate the inhibition efficiency of PBSLH using the following:

$$
I E_{\mathrm{I}}=\frac{R_{c t}-R_{c t}^{\prime}}{R_{c t}} \times 100
$$

where $R_{c t}^{\prime}$ and $R_{c t}$ were charge transfer resistance in the absence and presence of PBSLH. The double layer capacitance was obtained from the maximum value of the imaginary component of the Nyquist plots. Electrical equivalent circuit (EEC) was developed and used to explain the inhibitory action of PBSLH.

2.5. Surface Analysis. The scanning electron microscope (SEM) instrument TESCAN VEGA 3 was used to examine the copolymer and mild steel surface before and after the immersion in the electrolyte. The secondary electron detector was used to examine the surface morphology of PBSLH and mild steel. The EDS was also performed to determine the elemental composition of the composite. Moreover, atomic force microscope (AFM) was utilized to estimate the average roughness on the mild steel coupons.

\section{Results and Discussion}

3.1. Characterization of PBSLH. Figure 1 demonstrates the FTIR spectra of PBS, L-histidine and PBSLH. Every one of the three materials exhibits the characteristic vibrations of $\mathrm{N}$ $\mathrm{H}, \mathrm{C}-\mathrm{H}, \mathrm{C}=\mathrm{O}, \mathrm{C}-\mathrm{O}, \mathrm{C}-\mathrm{N}$, and $\mathrm{C}=\mathrm{N}$ while L-histidine showed additional vibration characteristic of $\mathrm{O}-\mathrm{H}$. The presence of these functional groups within the polymer composite confirms copolymerization of PBS and L-histidine. The wavenumbers at which these functional groups appeared differ in magnitude and intensities. This was because different strengths are on the bonds with each material. The major vibrational bonds in PBSLH were observed to appear at high energy.

The $\mathrm{C}=\mathrm{O}$ vibrational bands were observed for PBS, Lhistidine and PBSLH at 1712, 1628, and $1693 \mathrm{~cm}^{-1}$, respectively. The appearance nature of carbonyl bond in PBSLH had weak vibrational intensity and compared to PBS and L-histidine. These were because of the hydrogen bonding that took place between carbonyl bonds and $\mathrm{N}-\mathrm{H}$ during copolymerization. Because of the hydrogen bonding $\mathrm{C}=\mathrm{O}$ appeared at $25 \mathrm{~cm}^{-1}$ lower than the expected vibrational band; this was also confirmed by $[17,18]$. N-H symmetric stretching band was observed at $3191 \mathrm{~cm}^{-1}$ and its asymmetric stretch was observed at $1582 \mathrm{~cm}^{-1}$. C-H symmetric and asymmetric vibrations are observed at 2972 and $2856 \mathrm{~cm}^{-1}$,

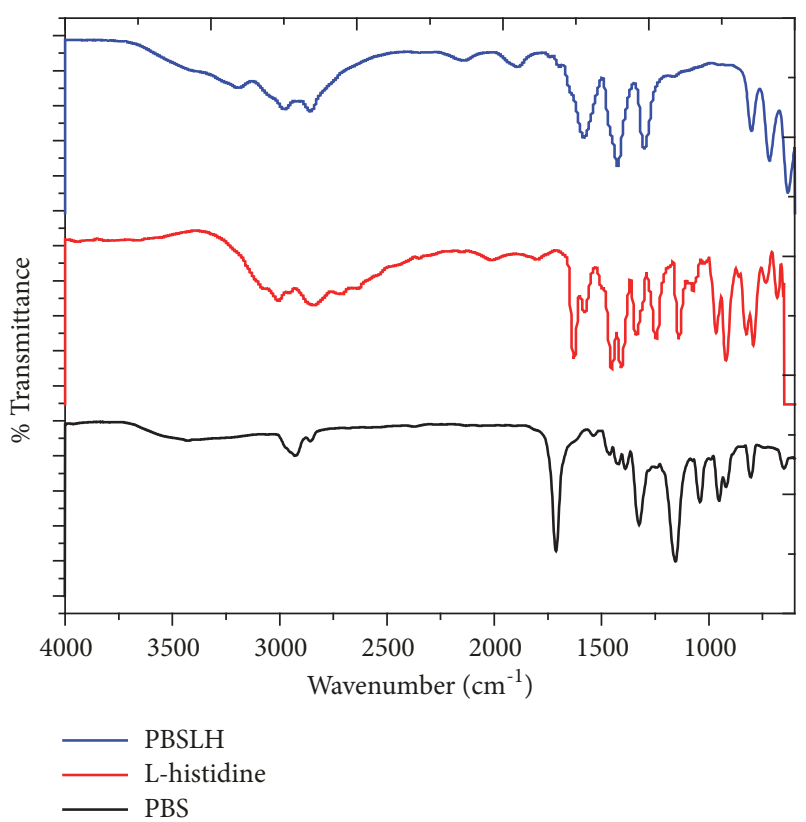

FIGURE 1: FTIR spectra of PBSLH, L-histidine, and PBS.

respectively, and its distortions were observed at 1423, 801, 717, and $631 \mathrm{~cm}^{-1}$, respectively.

As the affirmation of the presence of carboxylate group within the composite, $\mathrm{C}-\mathrm{O}$ vibration was observed at 1300 $\mathrm{cm}^{-1}$. Carboxylate group presence within the polymer composite indicated that PBS matrix did not break down during copolymerization; rather the composite was formed through hydrogen bonding between PBS and polymerized histidine. 2142 and $1892 \mathrm{~cm}^{-1}$ bands are attributed to $\mathrm{C}=\mathrm{N}$ and $\mathrm{C}=\mathrm{C}$ bonds, respectively, from imidazole ring of polymerized histidine.

The XRD was likewise used to confirm the polymerization; the XRD patterns of PBS and PBSLH are presented in Figure 2. The XRD diffractogram of PBS demonstrated the polymer as semicrystalline by the presence of high and sharp intensity peaks at $2 \theta=19.91^{\circ}$ and $22.98^{\circ}$, respectively. The peaks were due to strong ethylene group $\left(-\mathrm{CH}_{2}{ }^{-} \mathrm{CH}_{2}^{-}\right)$ which result in PBS not dissolving in aqueous solutions. The diffractogram of PBSLH indicated no presence of ethylene group, sharp peaks were observed at $2 \theta=14^{\circ}, 17$, and other peaks were observed between $34.72^{\circ}$ and $37.95^{\circ}$. These peaks were due to hydrogen bonding that happened between carbonyl of PBS and amine hydrogen from semipolymerized L-histidine. In Figure 2, the ethylene peak in PBS had been suppressed and the resulting polymer composite was soluble in aqueous solution.

The surface morphology of the copolymers was examined with SEM which is coupled with EDX (oxford energy dispersive). Distinctive phases that were found in the polymer composite were analyzed. The two phases obtained are shown in Figure 3(b), phase (i) was attributed to polymerized Lhistidine, and phase (ii) was attributed to PBS matrix. Figures 3(a) and 3(b) shows the micrographs of PBS and PBSLH, respectively, and two phases are observed in the polymer 


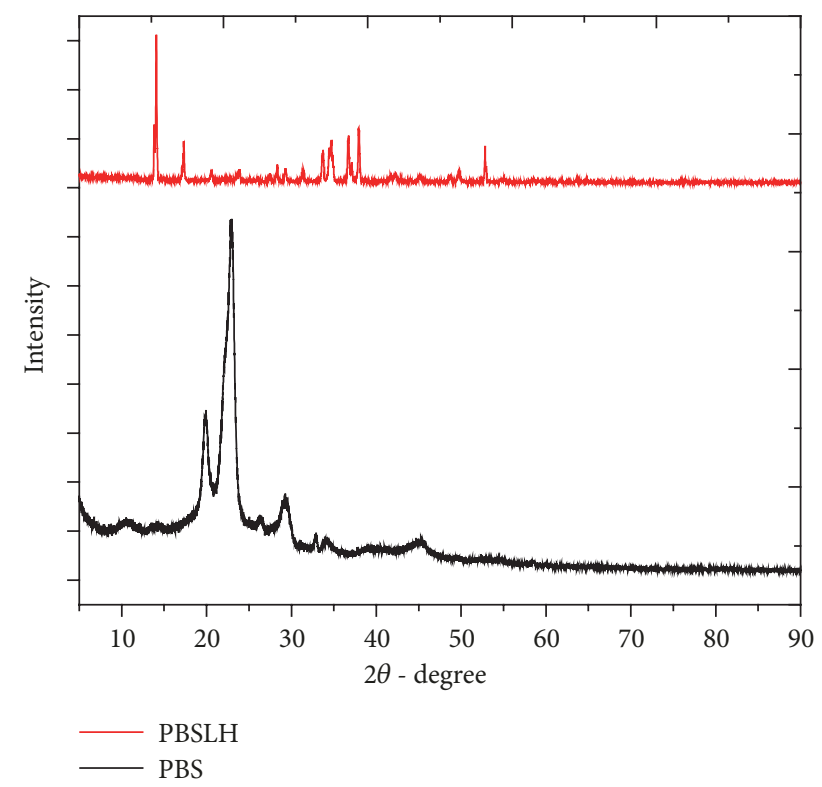

FIGURE 2: XRD pattern of PBSLH and PBS.

composite and one phase was observed in PBS polymer. The two phases observed; the stalk-like structures were due to PBS matrix and the thin rod-like structures were due to semipolymerized L-histidine.

To confirm these findings, SEM-EDX of the polymerized composite was performed and the \% count of elements was used to differentiate between PBS matrix and semipolymerized L-histidine. The SEM-EDX of PBS was also performed in order to form a basis of analysis. It was observed that a PBS polymer composite (PBSLH) contains the high percentage of $\mathrm{N}$ atom count [17]; from these results and pictures it can be concluded that the polymer composites had been produced. Moreover, the morphology of PBS and its composites were completely different; the difference in morphology was brought by semipolymerized L-histidine which was randomly distributed within the PBS polymer matrix and they were by hydrogen bonding.

\subsection{Gravimetric Analysis}

3.2.1. Effect of PBSLH Concentration. The dissolution of MS in acidic media for many years had been shown in the form of electrochemical reaction [19]. All these reactions including the high solubility of corrosion products result in the mass loss of mild steel. The corrosion inhibition performance of PBSLH against uniform corrosion was investigated by monitoring the weight loss as the process took place. The inhibition efficiencies were calculated from the corrosion rates as indicated by (1) and (2), while surface coverage was calculated by (3). In brief, 1000ppm solution of PBSLH in $1 \mathrm{M} \mathrm{HCl}$ was used as stock solution. The weight loss of MS was determined in the absence and presence of PBSLH. Figure 4 shows the variation of concentration of PBSLH with IE. The IE increases with the increase in concentration of the composite because of the availability of large number of adsorption sites that are brought by many macromolecule concentrations.

The maximum IE of $78 \%$ (PBSLH) was obtained at the optimum concentration of $600 \mathrm{ppm}$ attributed to the availability of $\mathrm{N}$ and $\mathrm{O}$ in the polymer composite which participates in the inhibitory action as active centers and electrostatic forces between PBSLH with mild steel. These heterogeneous atoms interact with the metal surface by adsorption hence increasing IE. Beyond this concentration the IE was observed to trop; this behavior was attributed to the saturation of polymer composites within the corrosive media, thereby reducing the interaction of the polymer composite with the metal surface resulting in reduced IE. The results clearly indicated that composting PBS with Lhistidine increases the solubility of the composite which in turn reduces the corrosion rate of mild steel. The active functional groups in the macromolecule which are carbonyl, amide, and ether groups interact with the surface of MS through electron donation to the d-orbital of $\mathrm{Fe}$ thereby enabling the adsorption of the copolymer to the mild steel surface.

3.2.2. Effect of Immersion Time. Time is a standout factor when characterizing the corrosion inhibitor. To determine the stability of the inhibitor film and rate of inhibitor adsorption, immersion time studies were conducted. The immersion periods were varied from 3 to 24 hours. The results are shown in Figure 4, which indicated the effect of immersion time on IE of PBSLH. The maximum corrosion inhibition efficiency was attained at the early period of immersion which was 3 hours. This was due to the quick adsorption of the inhibitor on to mild steel surface because there were maximum numbers of available active inhibitor molecules to inhibit mild steel from corroding. After 6 hours of immersion the IE was observed to decrease; it further decreased after the longest period of immersion which was chosen to be 24 hours in this study. The decrease in IE was attributed to desorption of the corrosion inhibitors from MS surface. Moreover, the corrosion rate (CR) also increased rapidly with a longer period of immersion. These happened because when PBSLH was being desorbed from MS surface, a bare metal was exposed to corrosive media thereby increasing metalsolution contact that led to more metals being dissolved. Moreover, the increase in CR after long period of immersion can also be attributed to a less concentration or limited number of available inhibitor polymer composites in $\mathrm{HCl}$ solution to stop or reduce metal dissolution; it is evident that after polymer composites had been desorbed from the metal surface they become inactive and therefore did not take part in the inhibition process. Owing to the composite biodegradability, it would bring no or major thread to the environment after its disposal.

3.2.3. Effect of Temperature. Weight loss measurements were also used to study the influence of temperature on the corrosion of MS with the presence of PBSLH corrosion inhibitor. The results are shown in Figure 5. The results were used to calculate activation energy of metal dissolution 


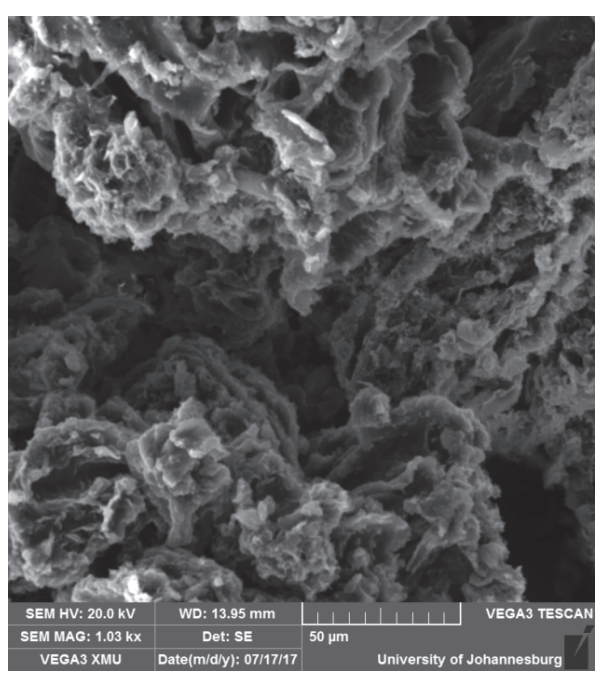

(a)

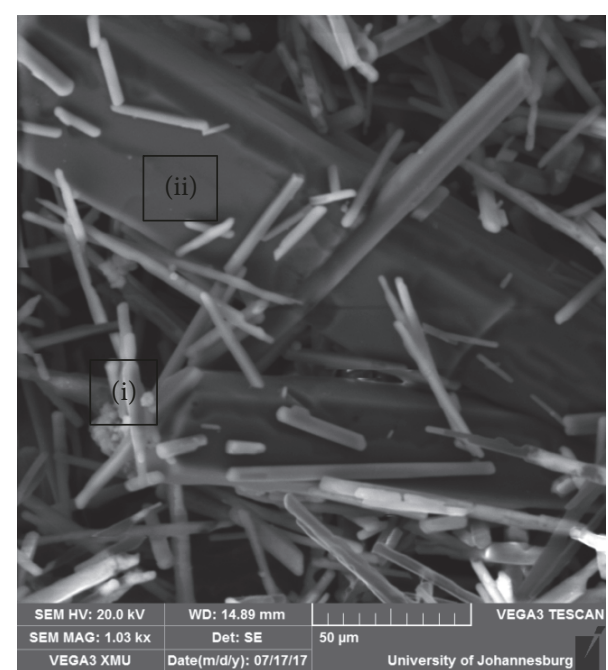

(b)

FIGURE 3: SEM micrograph of (a) PBS and (b) PBSLH.

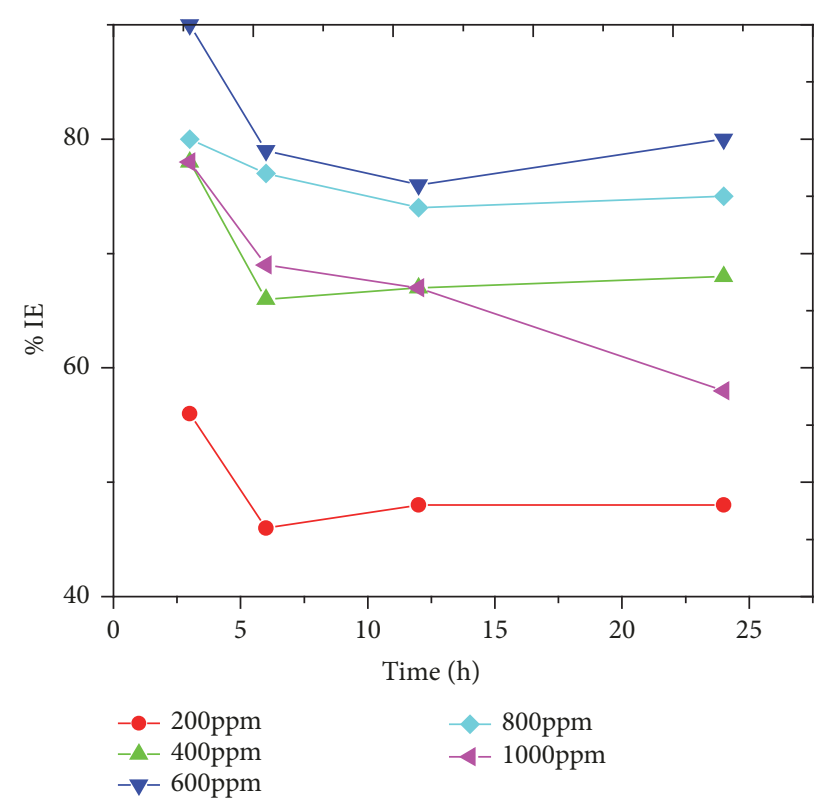

FIGURE 4: Effect of immersion time of mild steel in $1 \mathrm{M} \mathrm{HCl}$ in the absence and presence of different concentrations of PBSLH.

and Gibbs energy of adsorption of the inhibitor onto the mild steel surface. The IE was observed to decrease rapidly after with the increase of temperature. The IE obtained at optimum concentration was $78 \%$ at $298 \mathrm{~K}$ and $45 \%$ at $333 \mathrm{~K}$ in which the minimum and maximum temperatures were, respectively, utilized in this work. The decrease in IE of PBSLH at these temperatures showed the instability of PBSLH when it was subjected to higher temperatures. At higher temperatures PBSLH was desorbed from mild surface which implied that the adsorption of PBSLH to MS surface was an exothermic process, thereby realizing the heat during adsorption. According to Le Chatelier's principle when the

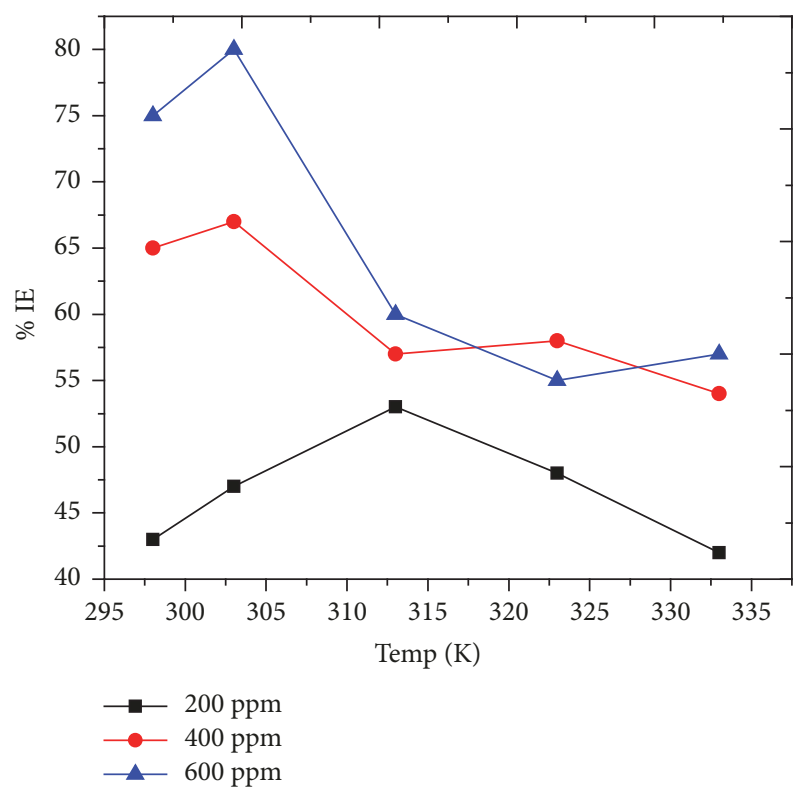

Figure 5: Effect of temperature on IE of PBSLH in $1 \mathrm{M} \mathrm{HCl}$.

reaction occurring is exothermic, the increase in temperature will cause the reverse of the reaction. The opposite is also true with endothermic reactions [20]. PBSLH had been found to work properly at lower temperatures with poor inhibition capabilities at higher temperatures. It can also be observed from Table 1 that when the temperature was increased, the corrosion rate was also increased.

3.2.4. Adsorption Isotherms. The adsorption isotherms provide information about the interaction of adsorbed molecules not only among themselves but also their interactions with a metal surface $[21,22]$. The adsorption of PBSLH macromolecules on to the mild steel surface is the imperative 
TABLE 1: Effect of temperature on the corrosion rate of MS in the absence and presence of PBSLH.

\begin{tabular}{lccccc}
\hline \multirow{2}{*}{ Temperature / K } & \multicolumn{3}{c}{$\begin{array}{c}\text { Corrosion rate }(\mathrm{CR}) / \mathrm{mg} \mathrm{cm}^{-2} \mathrm{~h}^{-1} \\
\text { Concentration / ppm (PBSLH) }\end{array}$} \\
\hline 298 & Blank & 200 & 400 & 600 \\
\hline 303 & 0.0548 & 0.0333 & 0.0215 & 0.0121 \\
\hline 313 & 0.0673 & 0.0410 & 0.0268 & 0.0182 \\
\hline 323 & 0.0790 & 0.0573 & 0.0349 & 0.0283 \\
\hline 333 & 0.1022 & 0.0844 & 0.0584 & 0.0402 \\
\hline
\end{tabular}

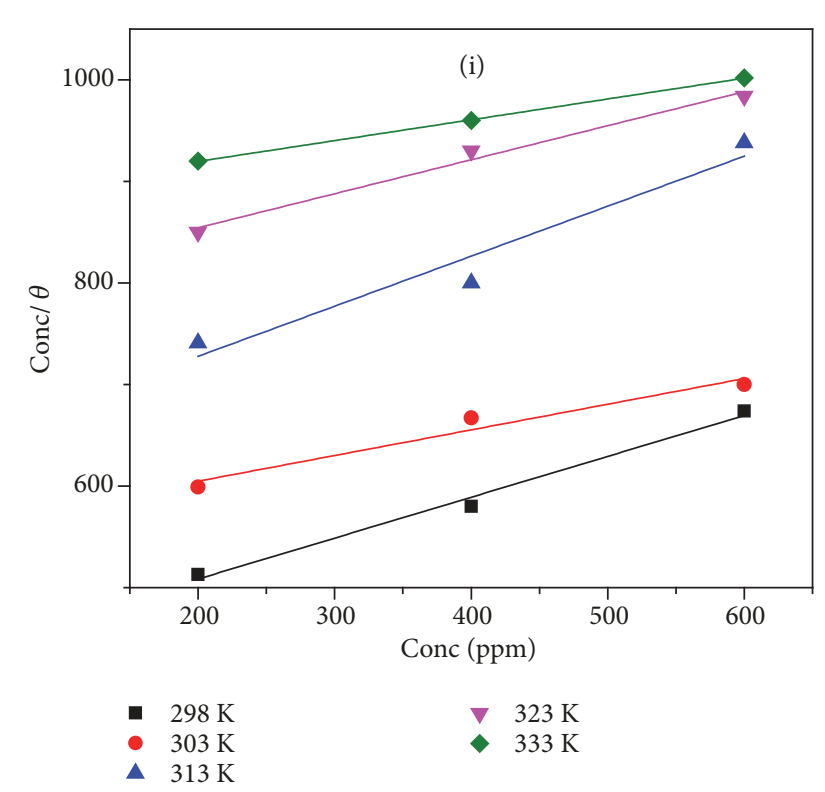

FIgURE 6: Langmuir plot for adsorption of PBSLH on to mild steel surface.

TABLE 2: Thermodynamic parameters for dissolution of MS in $1 \mathrm{M}$ $\mathrm{HCl}$ in the presesnce and absesnce of PBSLH.

\begin{tabular}{lccc}
\hline Conc/ppm & $\mathrm{E}_{\mathrm{a}} / \mathrm{KJmol}^{-1}$ & $\Delta \mathrm{H} / \mathrm{KJmol}^{-1}$ & $\Delta \mathrm{S} / \mathrm{KJmol}^{-1}$ \\
\hline Blank & 26.81 & 11.64 & -74.21 \\
\hline 200 & 32.53 & 14.13 & -164.39 \\
\hline 400 & 34.46 & 14.97 & -166.88 \\
\hline 600 & 65.10 & 28.27 & -182.59 \\
\hline
\end{tabular}

step of adsorption mechanism. The most essential parameter involved in this process is the surface coverage $(\theta)$ which can be calculated from gravimetric analysis as shown by (3).

Adsorption isotherm studies give the descriptive mechanism on how the organic inhibitor adsorb to the metal surface. This process is governed by various factors such as the nature and charge of the metal surface, adsorption of solvent and other ionic species, electronic characteristics of the metal surface, a temperature of corrosion reactants, and electronic potential of metal-solution interface $[23,24]$.

Adsorption of organic molecules occurs when the interaction energy between the metal surface and organic molecule is higher than that of metal surface and water molecule. During the study, Langmuir, Frumkin, and Parsons-Temkin adsorption models were tested and the model which gave the best fit was used to calculate thermodynamic parameters. From gravimetric results, it was concluded that the value of $\theta$ increases with the increase of concentration of PBSLH in $\mathrm{HCl}$; this was attributed to the formation film layer of PBSLH on to MS surface which decreases its dissolution rate of mild steel. Langmuir adsorption isotherm model was applied to investigate the adsorption mechanism. The following was used to calculate the equilibrium constant of adsorption:

$$
\frac{C}{\theta}=\frac{1}{K_{a d s}}+B
$$

where $\mathrm{C}$ is the inhibitor concentration (PBSLH) in $1 \mathrm{M} \mathrm{HCl}$, $\mathrm{K}_{\mathrm{ads}}$ is the equilibrium adsorption/desorption constant of PBSLH, and B is an intercept. Figure 6 presents Langmuir isotherm model plot for dissolution of mild steel which appeared to have correlation coefficients which were close to unity, which implied that the adsorption of the polymer composites on to mild surface assumed a mono layer adsorption; therefore based on the results it was concluded that adsorption of polymer composites on the mild steel surface obeyed Langmuir isotherm.

3.3. Thermodynamic and Kinetic Parameters. The kinetic parameters of the system under study were evaluated using the data obtained from gravimetric analysis for different concentrations of PBSLH composite at different temperatures. The apparent activation energy $(E a)$ of mild steel dissolution was calculated from Arrhenius equation described by the following relationship:

$$
\log C R=-\frac{E_{a}}{2.303 R T}+\log A
$$

where $\mathrm{CR}$ was the corrosion rate, $\mathrm{E}_{\mathrm{a}}$ was apparent activation energy, $\mathrm{R}$ was the molar gas constant, $\mathrm{A}$ was the frequency factor, and $\mathrm{T}$ was the absolute temperature. The plot of $\log C R$ vs $1 / \mathrm{T}$ in the presence and absence of different concentrations of PBSLH gave straight line graph shown in Figure 7 (i), the values of $E_{a}$ were calculated from the slope $\left(-E_{a} / 2.303 R\right)$, and their values were listed in Table 2 . The data showed that the activation energy of blank solution was 26.8 $\mathrm{KJmol}^{-1}$ which was less than the activation energy at 600 ppm concentration, $65.1 \mathrm{KJmol}^{-1}$. The increase in activation 

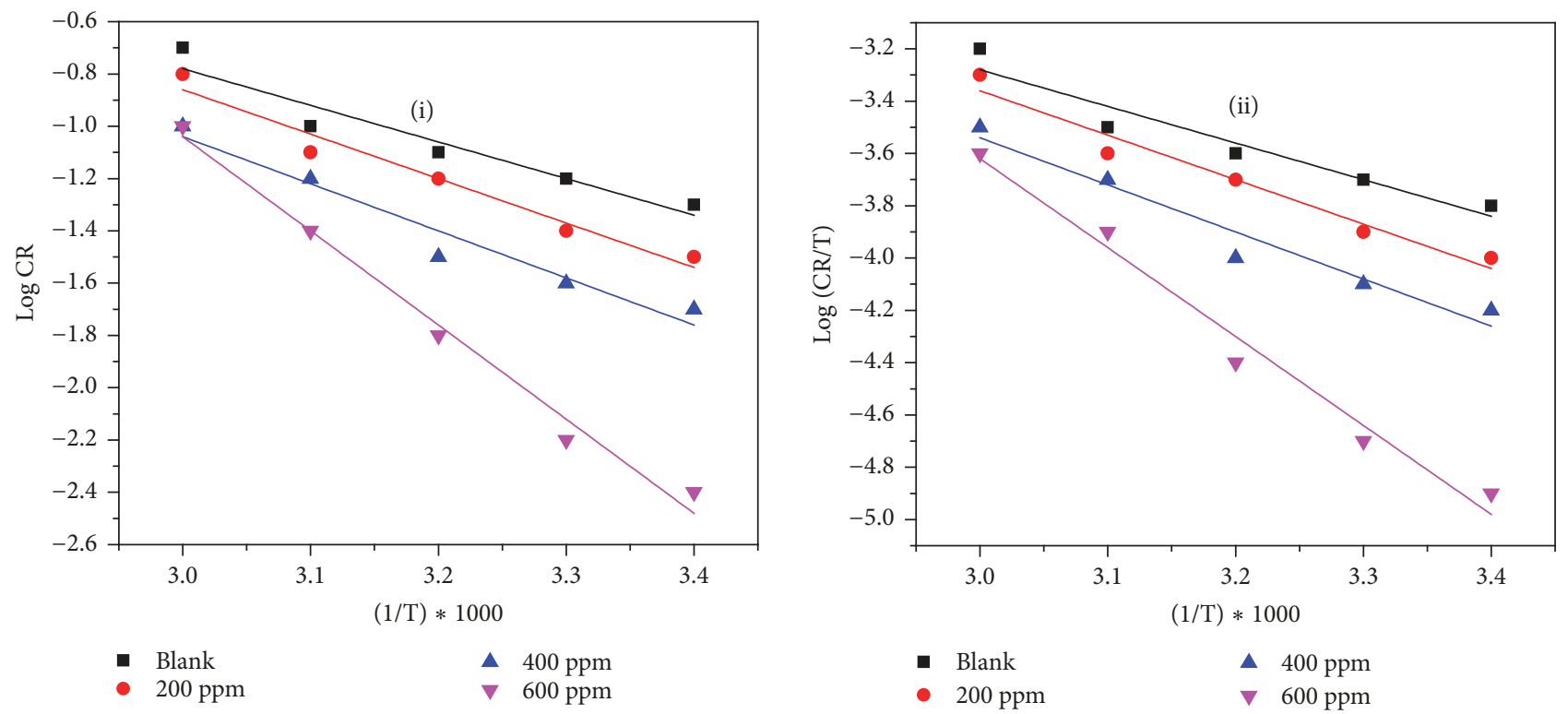

FIGURE 7: Arrhenius (i) and transition state (ii) plots for mild steel in $1 \mathrm{M} \mathrm{HCl}$ in the presence and absence of PBSLH of different concentration.

energy in the presence of optimum concentration results in the decrease of corrosion rate of mild steel hence high corrosion inhibition efficiency. This behavior was associated with the adsorption of PBSLH composite on the mild steel surface which decreases the surface area available for metal dissolution. Moreover, $\mathrm{E}_{\mathrm{a}}$ values increased with the increase in concentration which indicates that inhibition process is mainly controlled by activation energy [22]. Corrosion inhibition through physical adsorption of inhibitors had been associated with high activation energy of metal dissolution [25]; this happens because adsorbed inhibitors form a barrier at metal-solution interface which limits the contact of metal with corrosive medium. Therefore, for metal dissolution to take place in this scenario more apparent activation energy would be required for dissolution process to happen.

The increase in $E_{a}$ values suggests that the corrosion reaction would be further pushed towards the metal surface as the concentration of the inhibitor is further increased. Szauer and Brand (1981) explained that the increase in activation energy can be attributed to an appreciable decrease in the adsorption of the inhibitor on the mild steel surface with increase in temperature and a corresponding increase in corrosion rates since a greater area of metal is exposed to the acid environment.

The values of enthalpy $\left(\Delta \mathrm{H}_{\mathrm{a}}\right)$ and entropy $\left(\Delta \mathrm{S}_{\mathrm{a}}\right)$ of activation of MS dissolution were evaluated from the results of the effect of temperature on the process of dissolution using:

$$
\frac{\log C R}{T}=\log \left(\frac{R}{n h}\right)+\left(\frac{\Delta S_{a}}{2.303 R}\right)-\left(\frac{\Delta H_{a}}{2.303 T}\right)
$$

where $\mathrm{CR}$ was the corrosion rate at temperature $\mathrm{T}, \mathrm{R}$ was the gas molar constant, $n$ was Avogadro's constant, and $h$ was the Planck's constant. The plot of $\log (\mathrm{CR} / \mathrm{T})$ vs $1 / \mathrm{T}$ Figure 7 (ii) for different corrosion system yielded straight line curves.
The slope of the curves corresponds to $(-\Delta \mathrm{H} / 2.303 \mathrm{R})$ and the intercept to $\log (\mathrm{R} / n h)+\Delta \mathrm{S} / 2.303 \mathrm{R}$; these were used to calculate $\Delta \mathrm{H}_{\mathrm{a}}$ and $\Delta \mathrm{S}_{\mathrm{a}}$ presented in Table 2 .

The positive values of $\Delta \mathrm{H}$ indicate that the MS dissolution was an endothermic process suggesting that dissolution of MS was slow at low temperatures in the presence of the polymer composite as a corrosion inhibitor. When the temperature was further increased the values of $\Delta \mathrm{H}$ were also increasing confirming the endothermic nature of mild steel dissolution and inhibition efficiency of PBSLH composites was decreased. The values of entropy of activation $\left(\Delta \mathrm{S}_{\mathrm{a}}\right)$ were obtained to be largely negative at lower temperatures but as temperature was further increased they were becoming more positive. Moreover, without the presence of inhibitor $\Delta \mathrm{S}_{\mathrm{a}}$ was largely positive and tends to be more negative with the increase of the concentration of inhibitors. Negative activated entropy indicated that, at lower temperatures and higher concentrations, the surface of mild steel was largely covered by the composites; therefore there was less metal dissolution (disorder) taking place. The dissolution of mild was prevented by the barrier layer that was formed between metal-solution interfaces. At higher temperatures, metal dissolution was rapid due to the damaged or removed barrier layer that was due to adsorbed polymer composites; hence the values of $\Delta \mathrm{S}_{\mathrm{a}}$ were more positive at higher temperatures as compared to lower temperatures which confirms the high disorder of mild steel surface. This indicates that the system passes to a more random arrangement.

3.4. Morphological Studies. The surface of MS was analyzed with SEM before and immersion in $1 \mathrm{M} \mathrm{HCl}$ (blank solution) and 600 ppm PBSLH solution. The immersion period was 3 hours. Figure 8 shows the micrographs of mild steel and it can be observed that MS in blank solution corroded more than other specimens; this is because PBSLH inhibitor was 


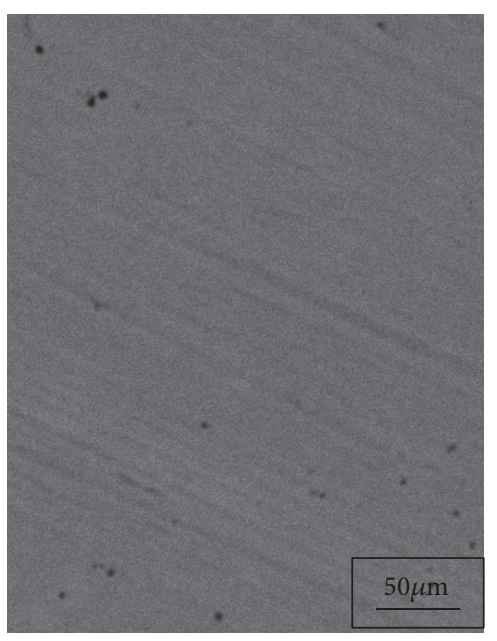

(a)

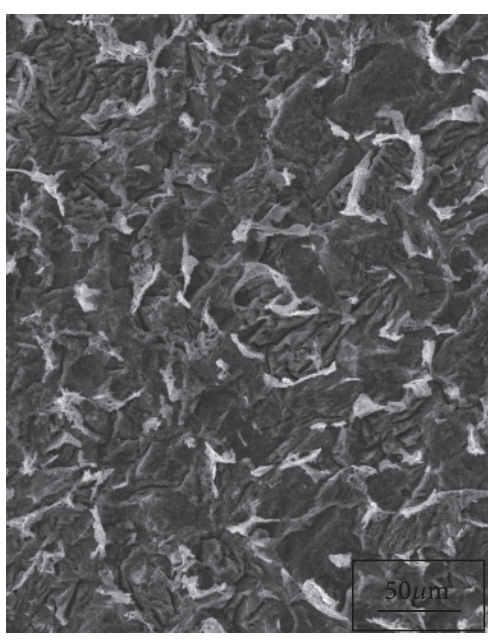

(b)

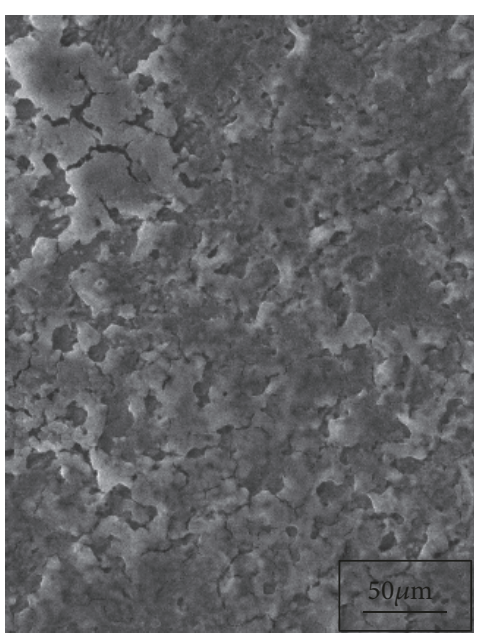

(c)

FIGURE 8: Micrographs of mild steel surface (a) before immersion in $\mathrm{HCl}$ (b) After immersion in $1 \mathrm{M} \mathrm{HCl}$ (blank) (c) After immersion in 1 $\mathrm{M} \mathrm{HCl}$ with 600 ppm PBSLH.

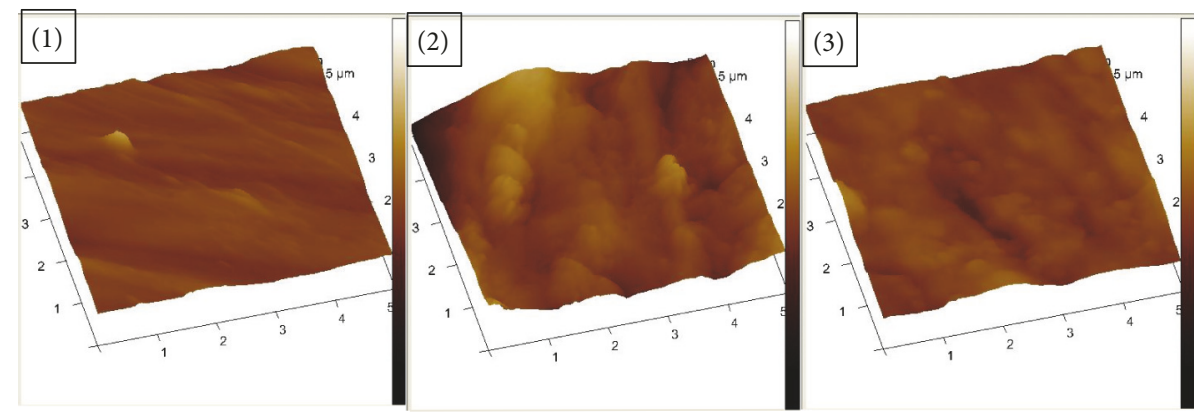

FIGURE 9: AFM micrographs of mild steel (1) unaffected, (2) blank, and (3) 600 ppm PBSLH.

effective in reducing the dissolution of mild steel. Moreover, AFM was used to characterize the average roughness of mild steel before and after exposure to $\mathrm{HCl}$. The AFM micrographs Figure 9 showed that the mild steel coupon that was immersed in the 600 ppm PBSLH solution of low average roughness compared to the one that was exposed to the blank solution. It was concluded with the observed results that PBSLH can reduce the corrosion rate of mild steel in $1 \mathrm{M}$ $\mathrm{HCl}$.

\subsection{Electrochemical Analysis}

3.5.1. Potentiodynamic Polarization (Tafel Plots). The potentiodynamic polarization curves of the mild steel in $1 \mathrm{M}$ $\mathrm{HCl}$ solution are with various concentrations of polymer composite; PBSLH after $2 \mathrm{~h}$ of immersion time are presented in Figure 10. It should be noted that prior the experiments were carried out, OCP was established. All potential measurements were measured with respect to Saturated Calomel Electrode (SCE) in an open circuit potential. It was evident from the plots that addition of both PBSLH composites suppressed both cathodic and anodic reaction reducing hydrogen evolution and dissolution of mild steel; this was in conjunction with results obtained in gravimetric analysis.

The related electrochemical parameters such as corrosion potential $\left(\mathrm{E}_{\text {corr }}\right)$ and corrosion current density $\left(\mathrm{i}_{\text {corr }}\right)$ were obtained from Tafel plots which were calculated directly from the EC-Lab software by Tafel fit. The IE was calculated from polarization measurements according to equation (4) and results are shown in Table 3. The value of $\mathrm{I}_{\text {corr }}$ decreased with the increase in the concentration of the inhibitor PBSLH as shown in Table 3; this was due to the adsorption of the polymer composite to the surface of mild steel which limits the dissolution of mild steel thereby reducing corrosion rate.

In addition, from Table 2 the value of $\beta_{a}$ changes with the increase of concentration of both polymer composites and more pronounced changes were observed with $\beta_{c}$; this indicated that both anodic and cathodic reactions were affected but the effect was more prominent on cathodic reaction. The shift in anodic Tafel slope $\beta_{a}$ was due to adsorption of inhibitor molecules on to the mild steel surface (anodic sites) [26]. Generally, an inhibitor can be classified as cathodic or anodic inhibitor if the shift in $\mathrm{E}_{\text {corr }}$ in the presence of inhibitor is greater than $85 \mathrm{mV}$ with respect to $\mathrm{E}_{\text {corr }}$ of blank 
TABLE 3: Electrochemical parameters for corrosion MS in different concentrations of PBSLH.

\begin{tabular}{lcccccccc}
\hline Conc/ppm & $\mathrm{E}_{\mathrm{corr}} / \mathrm{mV}$ & $\mathrm{I}_{\text {corr }} / \mathrm{mAcm}^{-2}$ & $\beta_{\mathrm{a}} / \mathrm{mV}$ & $\beta_{\mathrm{c}} / \mathrm{mV}$ & \multicolumn{2}{c}{$\mathrm{CR} / \mathrm{mmpy}$} & $\Theta$ & $\% \mathrm{IE}_{\mathrm{p}}$ \\
\hline Blank & -527.57 & 6.79 & 474.2 & 531.2 & 77.81 & - \\
\hline 200 & -520.69 & 2.12 & 216.6 & 302.7 & 24.34 & 0.69 & 69 \\
\hline 400 & -522.70 & 1.81 & 282.7 & 333.0 & 20.60 & 0.73 & 73 \\
\hline 600 & -514.59 & 1.49 & 228.9 & 280.5 & 17.48 & 0.78 & 78 \\
\hline
\end{tabular}

TABLE 4: VASP parameters for corrosion of MS in $1 \mathrm{M} \mathrm{HCl}$ with different concentrations of PBSLH.

\begin{tabular}{|c|c|c|c|c|c|}
\hline Conc/ppm & $\mathrm{I}_{\text {corr }} / \mathrm{mA}$ & $\beta_{\mathrm{a}} / \mathrm{mV}$ & $\beta_{\mathrm{c}} / \mathrm{mV}$ & $\mathrm{R}_{\mathrm{p}} / \mathrm{Ohms}$ & $\% \mathrm{IE}_{\mathrm{v}}$ \\
\hline Blank & $10.34 \mathrm{e} 6$ & 29711 & 51490 & 0.20 & - \\
\hline 200 & $8.666 \mathrm{e} 6$ & 44655 & 466903 & 2.25 & 91 \\
\hline 400 & $5.624 \mathrm{e} 6$ & 37357 & 549408 & 2.50 & 92 \\
\hline 600 & $5.848 \mathrm{e} 6$ & 34878 & 560502 & 2.78 & 93 \\
\hline
\end{tabular}

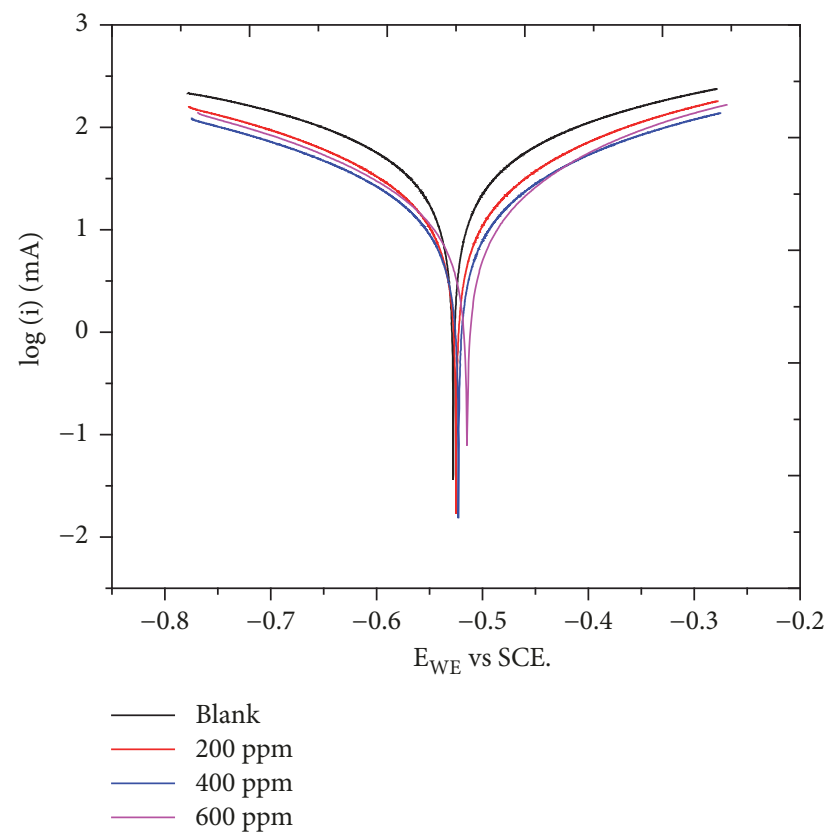

Figure 10: Tafel plot for corrosion of mild steel in the absence and presence of different concentrations of PBSLH.

solution. Otherwise the inhibitor is termed a mixed type corrosion inhibitor [27], meaning both cathodic and anodic reactions are significantly affected by the inhibitor; therefore, PBSLH was termed a mixed type corrosion inhibitor. In the present study the maximum shift was $59.16 \mathrm{mV}$ anodically when compared to the blank. This showed that the corrosion rate of mild was predominately controlled by oxidation of Fe.

The maximum corrosion inhibition efficiency obtained in potentiodynamic polarization (PDP) for PBSLH composite was $78 \%$. As stated before, the same trend was observed with the results from gravimetric studies. This implied that polymer composite PBSLH inhibited corrosion upon its addition to corrosive media. The corrosion inhibition mechanism by PBSLH composite was through suppressing both cathodic and anodic reactions also by film forming on mild steel

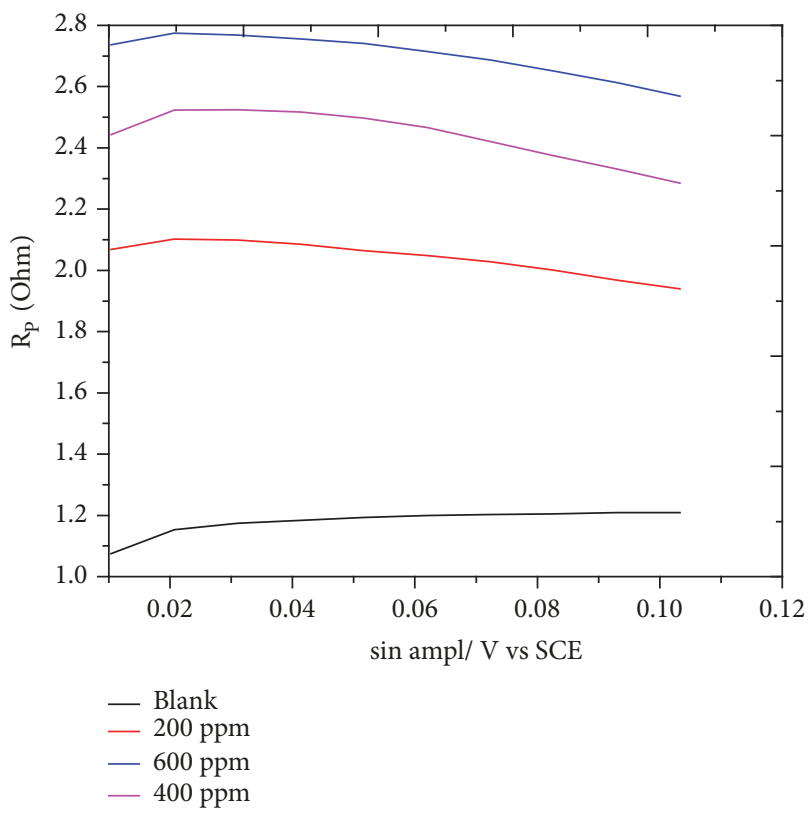

FIGURE 11: VASP plot for corrosion of mild steel in $1 \mathrm{M} \mathrm{HCl}$ in the absence and presence of PBSLH.

surface. Even though both redox reactions were suppressed, from the Tafel plots the cathodic side was the most affected; thus hydrogen evolution was reduced simultaneously controlling number of electrons released from anodic side. From the set of results, it was concluded that cathodic reaction was the rate determining step in the corrosion of mild steel.

3.5.2. VASP. VASP is the technique that offers the determination of polarization resistance $\left(\mathrm{R}_{\mathrm{p}}\right)$ and other corrosion parameters as they are listed in Table 4 . Figure 11 shows the plot of $R_{p}$ vs sinus amplitude for the dissolution of MS in 1 $\mathrm{M} \mathrm{HCl}$ with different concentration of PBSLH. In the case of corrosion of mild steel, the electric current was caused by dissolution of Fe from mild steel. Polarization may occur on either anodic or cathodic side depending on the dominance 


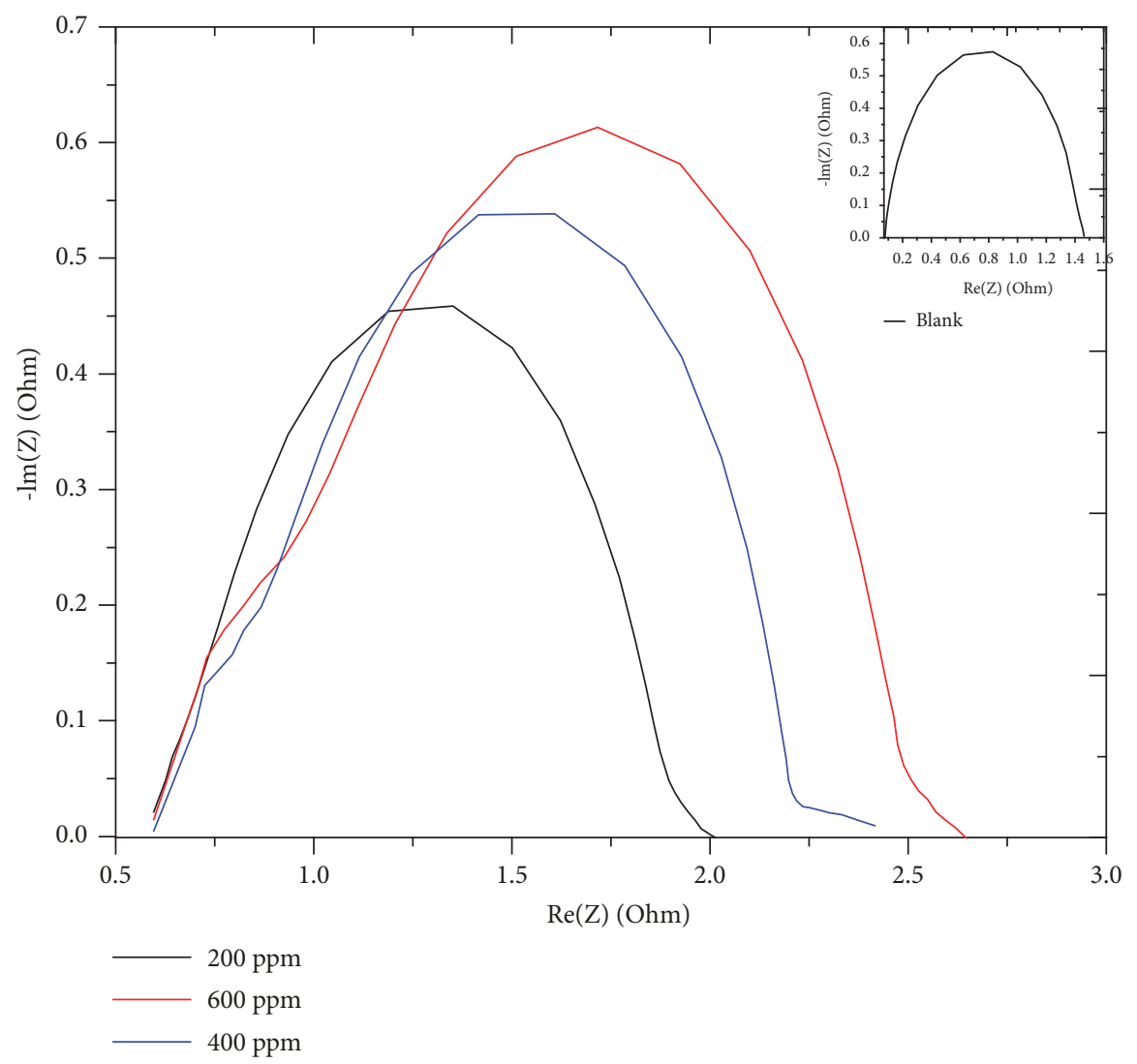

FIGURE 12: Nyquist plot for dissolution of mild steel in the presence and absence of PBSLH in $1 \mathrm{M} \mathrm{HCl}$.

of the reaction; since cathodic side was more dominant therefore mild steel was cathodically polarized.

From Figure 11, it can be observed that $R_{p}$ of the system increased with increasing concentration of the PBSLH inhibitor; this was caused by more film formation of the inhibitor on the mild steel surface as the concentration was increased. Increasing the concentration on the inhibitor provided more active sides for absorption; hence the insulation is increased by a film forming.

3.5.3. EIS. The results of impedance measurements were carried out in the wide frequency range of $100 \mathrm{~Hz}$ to $100 \mathrm{MHz}$, providing information related to the tested corrosion system and electrochemical process occurring. The interpretation of EIS measurement involves the use of electrode-equivalent circuits (EEC). The individual elements of EEC correspond to the electrochemical properties of the tested corrosion system [28], in this regard, $1 \mathrm{M} \mathrm{HCl}$ with and without the presence of polymer composites as corrosion inhibitors. This allowed the determination of corrosion parameters such as charge transfer resistance $\left(\mathrm{R}_{\mathrm{ct}}\right)$. Impedance modeling of a complex system requires the utilization of complex EEC systems with constant phase elements (CPE). CPE element has a fixed phase shift angle and its impedance describes by the following relation: $Z_{C p e}=1 / Y_{0}(j \omega)^{n}$, where $Y_{0}$ and $n$ are parameters related to the phase shift angle [29, 30].
The value of the parameter $\mathrm{n}$ describes the corrosion process on the surface of the material [31]; the smaller the value of these parameters, the heterogeneous process which is caused by the unavoidable results of polishing the mild steel surface [32]. The factor of $\mathrm{n}$ is adjustable parameter of values from -1 to 1 , where a value of -1 is the characteristic for an inductance, a value of 1 corresponds to a capacitor, and the value of 0 corresponds to a resistor $[30,33]$.

Figure 12 indicates the Nyquist plot for mild steel in different concentrations of PBSLH. From the results obtained, it is observed that the size of the semicircles increased with the increase of the concentration of the inhibitors. This was caused by the formation of a film layer on the surface of mild steel by the inhibitors; as the concentration was increased more inhibitor molecules were available giving more coverage on the mild steel surface at the high concentration hence larger semicircle.

The EEC was used to fit the experimental (impedance) data on the PBSLH corrosion inhibitor composite is represented in Figure 13. Element $\mathrm{R}_{1}$ corresponds to solution resistance $\left(R_{s}\right)$ which was a fixed value, while element $R_{2}$ corresponds to charge transfer resistance $\left(R_{c t}\right)$ in phase interface and is inversely proportional to corrosion rate and surface area undergoing corrosion. The CPE elements $\mathrm{C}_{1}$ and $\mathrm{C}_{3}$ characterize a capacitance depression related to capacity of the material surface area of a complex surface roughness, 
TABLE 5: Fitting EIS parameters for dissolution in the absence and presence of different concentration PBSLH.

\begin{tabular}{lcccccc}
\hline Conc/ppm & $\mathrm{R}_{1} / \mathrm{Ohm}$ & $\mathrm{C}_{1} \times 10^{-3} / \mathrm{F}$ & $\mathrm{R}_{2} / \mathrm{Ohm}$ & $\mathrm{C}_{3} / \mathrm{F}$ & $\mathrm{R}_{3} / \mathrm{Ohm}$ & $\% \mathrm{IE}_{\mathrm{E}}$ \\
\hline Blank & 1.199 & 2.784 & 0.0568 & $0.311 \mathrm{e} 15$ & $-2.77 \mathrm{e} 12$ & - \\
\hline 200 & 0.650 & 0.608 & 0.897 & $1.729 \mathrm{e}-3$ & 0.9416 & 94 \\
\hline 400 & 0.824 & 0.510 & 1.395 & $1.017 \mathrm{e}-3$ & 1.387 \\
\hline 600 & 0.811 & 0.723 & 1.496 & $0.205 \mathrm{e}-3$ & 0.285 & 95 \\
\hline
\end{tabular}

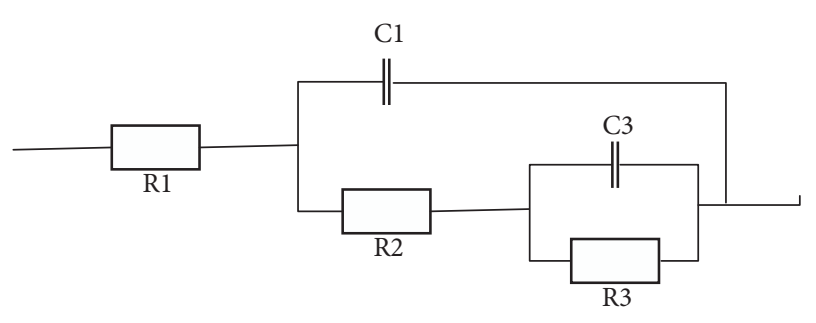

FIGURE 13: EEC used for modeling metal/solution interface.

inhomogeneous reaction rates on a surface, and nonuniform current distribution. Element $\mathrm{R}_{3}$ corresponds to electrolyte resistance present in porosity in this material zone. Equation (5) was used to calculate the IE, and the results are shown in Table 5. From Table 5, the values CPE decreased with the increase of the concentration of PBSLH; therefore it is concluded that the formation of film layer on the surface of mild steel reduces the interaction with the electrolyte because less charge was stored in the interface.

\section{Conclusions}

The corrosion inhibition of mild steel was studied by gravimetric, electrochemical, and morphological studies.

(1) The composite PBSLH inhibits corrosion of mild steel in $1 \mathrm{M} \mathrm{HCl}$ to a great extent and its ability was due to the presence of multiple $\mathrm{N}-\mathrm{H}$ and $\mathrm{C}=\mathrm{O}$ groups that act as the active centers of adsorption.

(2) The adsorbed film was stable at $30^{\circ} \mathrm{C}$.

(3) The potentiodynamic polarization methods indicated that PBSLH is a mixed type corrosion inhibitor.

(4) EIS measurements revealed the formation of barrier layer on mild steel surface.

(5) The absorption of PBSLH on to mild steel surface obeys Langmuir isotherm.

(6) SEM and AFM confirmed the inhibition performance of the PBSLH.

\section{Data Availability}

The data used to support the findings of this study are available from the corresponding author upon request.

\section{Conflicts of Interest}

The authors declare that there are no conflicts of interest regarding the publication of this paper.

\section{Acknowledgments}

The authors would like to thank NRF for funding the project.

\section{References}

[1] L. Hamadi, S. Mansouri, K. Oulmi, and A. Kareche, "The use of amino acids as corrosion inhibitors for metals: a review," Egyptian Journal of Petroleum, vol. 27, no. 4, pp. 1157-1165, 2018.

[2] A. A. L. I. F. Sabirneeza, R. Geethanjali, and S. Subhashini, "Polymeric corrosion inhibitors for iron and its alloys?: a review," Chemical Engineering Communications, vol. 202, no. 2, pp. 232-244, 2015.

[3] D. E. Arthur, A. Jonathan, P. O. Ameh, and C. Anya, "A review on the assessment of polymeric materials used as corrosion inhibitor of metals and alloys," International Journal of Industrial Chemistry, vol. 4, no. 1, p. 2, 2013.

[4] D. Karthik, D. Tamilvendan, and G. Venkatesa Prabhu, "Study on the inhibition of mild steel corrosion by 1,3-bis-(morpholin4-yl-phenyl-methyl)-thiourea in hydrochloric acid medium," Journal of Saudi Chemical Society, vol. 18, no. 6, pp. 835-844, 2014.

[5] S. A. A. El-Maksoud, "The effect of organic compounds on the electrochemical behaviour of steel in acidic media . a review," International Journal of Electrochemical Science, vol. 3, pp. 528$555,2008$.

[6] M. Finšgar and J. Jackson, "Application of corrosion inhibitors for steels in acidic media for the oil and gas industry: a review," Corrosion Science, vol. 86, pp. 17-41, 2014.

[7] B. E. A. Rani and B. B. J. Basu, "Green inhibitors for corrosion protection of metals and alloys: an overview," International Journal of Corrosion, vol. 2012, Article ID 380217, 15 pages, 2012.

[8] P. Preethi Kumari, S. Rao, and P. Shetty, "Corrosion inhibition of mild steel in $2 \mathrm{M} \mathrm{HCl}$ by a schiff base derivative," Procedia Materials Science, vol. 5, pp. 499-507, 2014.

[9] E. E. Ebenso, H. Alemu, S. A. Umoren, and I. B. Obot, "Inhibition of mild steel corrosion in sulphuric acid using alizarin yellow GG dye and synergistic iodide additive," International Journal of Electrochemical Science, vol. 3, no. 12, pp. 1325-1339, 2008.

[10] G. H. Koch, M. P. H. Brongers, N. G. Thompson, Y. P. Virmani, and J. H. Payer, Corrosion Cost and Preventetive Stratergies in the United states, United States: NACE International, CC Technologies Laboratory, 2002.

[11] T. L. Skovhus, R. B. Eckert, and E. Rodrigues, "Management and control of microbiologically influenced corrosion (MIC) in 
the oil and gas industry-Overview and a North Sea case study," Journal of Biotechnology, vol. 256, no. July, pp. 31-45, 2017.

[12] L. Adamczyk, "Electrochemical and anticorrosion properties of bilayer polyrhodanine / isobutyltriethoxysilane coatings," Journal of Applied Electrochemistry, 2016.

[13] M. O’Donoghue, R. Garrett, V. Datta, P. Roberts, and T. Aben, "EIS: testing coatings for rapid immersion service," Materials Performance, pp. 36-41, 2003.

[14] N. O. Eddy, "Experimental and theoretical studies on some amino acids and their potential activity as inhibitors for the corrosion of mild steel, part 2," Journal of Advanced Research, vol. 2, no. 1, pp. 35-47, 2011.

[15] N. El Hamdani, R. Fdil, M. Tourabi, C. Jama, and F. Bentiss, "Alkaloids extract of Retama monosperma (L.) Boiss. seeds used as novel eco-friendly inhibitor for carbon steel corrosion in 1 $\mathrm{M} \mathrm{HCl}$ solution: electrochemical and surface studies," Applied Surface Science, vol. 357, pp. 1294-1305, 2015.

[16] K. C. Emregül, R. Kurtaran, and O. Atakol, "An investigation of chloride-substituted Schiff bases as corrosion inhibitors for steel," Corrosion Science, vol. 45, no. 12, pp. 2803-2817, 2003.

[17] A. A. Fathima, S. S. Subhashini, and Á. P. Á. Impedance, "Poly (vinyl alcohol proline) as corrosion inhibitor for mild steel in $1 \mathrm{M}$ hydrochloric acid," International Journal of Industrial Chemistry, vol. 5, pp. 111-120, 2014.

[18] M. Prabakaran, S. Vadivus, S. Ramesh, and V. Periasamy, "Corrosion protection of mild steel by a new phosphonate inhibitor system in aqueous solution," Egyptian Journal of Petroleum, vol. 23, no. 4, pp. 367-377, 2014.

[19] M. Benabdellah, K. F. Khaled, and B. Hammouti, "Kinetic investigation of C38 steel corrosion in concentrated perchloric acid solutions," Materials Chemistry and Physics, vol. 120, no. 1, pp. 61-64, 2010.

[20] P. Atkins and J. De Paula, Physical Chemistry, W.H. Freeman and Company, New York, NY, USA, 9th edition, 2010.

[21] A. A. F. Sabirneeza, "A novel water-soluble, conducting polymer composite for mild steel acid corrosion inhibition," Journal of Applied Polymer Science, vol. 127, no. 4, pp. 3084-3092, 2016.

[22] A. Biswas, S. Pal, and G. Udayabhanu, "Experimental and theoretical studies of xanthan gum and its graft co-polymer as corrosion inhibitor for mild steel in 15\% HCl," Applied Surface Science, vol. 353, pp. 173-183, 2015.

[23] I. A. Adejoro, F. K. Ojo, and S. K. Obafemi, "Corrosion inhibition potentials of ampicillin for mild steel in hydrochloric acid solution," Journal of Taibah University for Science, vol. 9, no. 2, pp. 196-202, 2015.

[24] N. O. Obi-Egbedi, I. B. Obot, and S. A. Umoren, "Spondias mombin L. as a green corrosion inhibitor for aluminium in sulphuric acid: correlation between inhibitive effect and electronic properties of extracts major constituents using density functional theory," Arabian Journal of Chemistry, vol. 5, no. 3, pp. 361-373, 2012.

[25] K. K. Alaneme, S. J. Olusegun, and A. W. Alo, "Corrosion inhibitory properties of elephant grass (Pennisetum purpureum) extract: effect on mild steel corrosion in $1 \mathrm{M} \mathrm{HCl}$ solution," Alexandria Engineering Journal, vol. 55, no. 2, pp. 1069-1076, 2016.

[26] P. Muthukrishnan, P. Prakash, B. Jeyaprabha, and K. Shankar, "Stigmasterol extracted from Ficus hispida leaves as a green inhibitor for the mild steel corrosion in $1 \mathrm{M} \mathrm{HCl}$ solution," Arabian Journal of Chemistry, 2015.
[27] C. B. Pradeep Kumar and K. N. Mohana, "Phytochemical screening and corrosion inhibitive behavior of Pterolobium hexapetalum and Celosia argentea plant extracts on mild steel in industrial water medium," Egyptian Journal of Petroleum, vol. 23, no. 2, pp. 201-211, 2014.

[28] J. R. Macdonald, "Impedance spectroscopy," Annals of Biomedical Engineering, vol. 20, no. 3, pp. 289-305, 1992.

[29] Z. Brytan, J. Niagaj, and Ł. Reiman, "Applied Surface Science Corrosion studies using potentiodynamic and EIS electrochemical techniques of welded lean duplex stainless steel UNS S82441," Applied Surface Science, vol. 388, pp. 160-168, 2016.

[30] M. Behpour, S. M. Ghoreishi, and N. Soltani, "The inhibitive effect of some bis-N, S-bidentate Schiff bases on corrosion behaviour of 304 stainless steel in hydrochloric acid solution PA," Corrosion Science, vol. 51, no. 5, pp. 1073-1082, 2009.

[31] A. Döner, R. Solmaz, M. Özcan, and G. Kardaş, "Experimental and theoretical studies of thiazoles as corrosion inhibitors for mild steel in sulphuric acid solution," Corrosion Science, vol. 53, no. 9, pp. 2902-2913, 2011.

[32] E. E. Elemike, H. U. Nwankwo, D. C. Onwudiwe, and E. C. Hosten, "Synthesis, structures, spectral properties and DFT quantum chemical calculations of (E)-4((4-propylphenyl)imino)methyl)phenol and (E)-4-((2tolylimino)methyl)phenol; their corrosion inhibition studies of mild steel in aqueous HCl," Journal of Molecular Structure, vol. 1141, pp. 12-22, 2017.

[33] J. R. Macdonald and L. D. Potter, "A flexible procedure for analyzing impedance spectroscopy results: description and illustrations," Solid State Ionics, vol. 24, no. 1, pp. 61-79, 1987. 


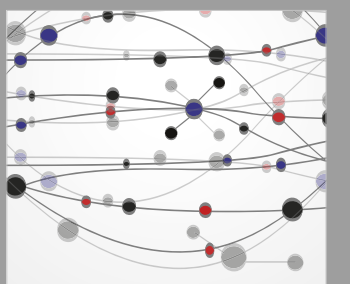

The Scientific World Journal
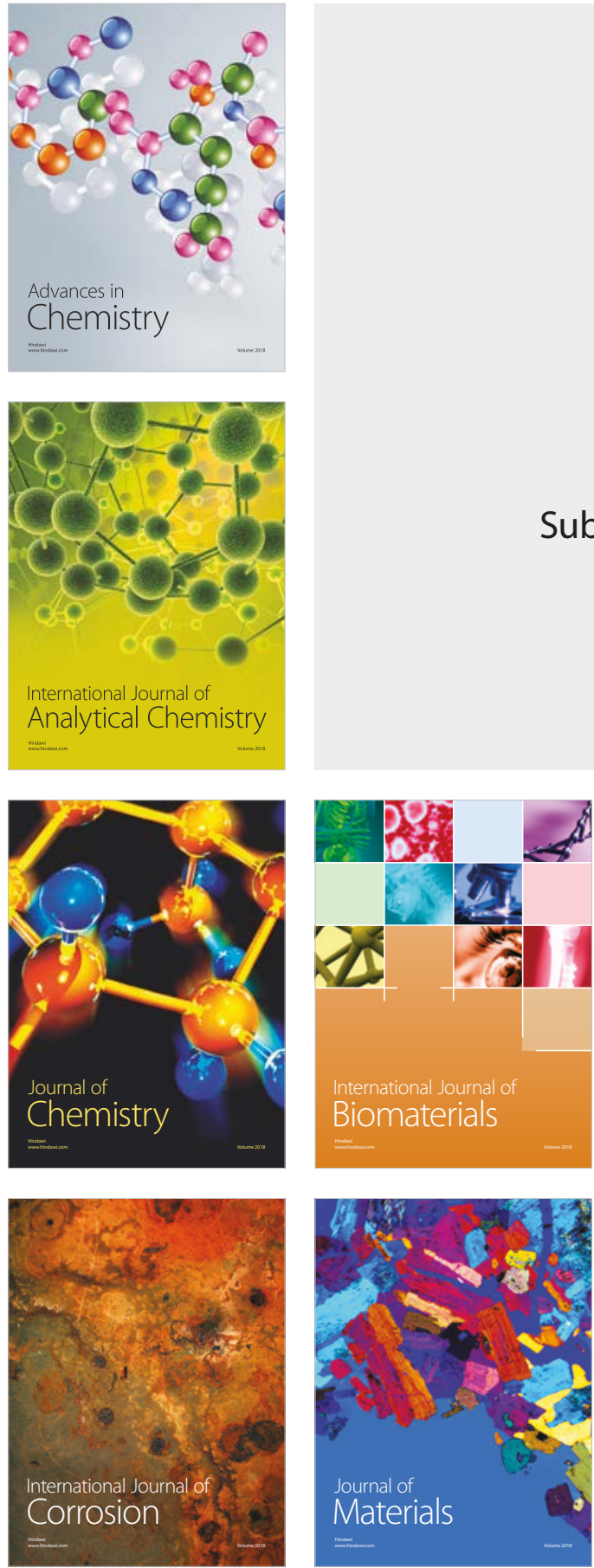

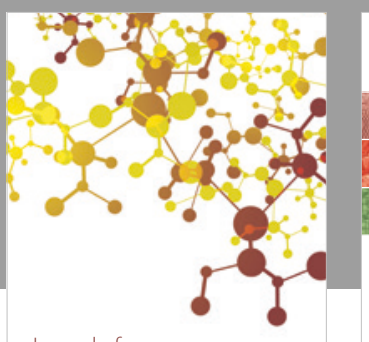

Journal of

Applied Chemistry
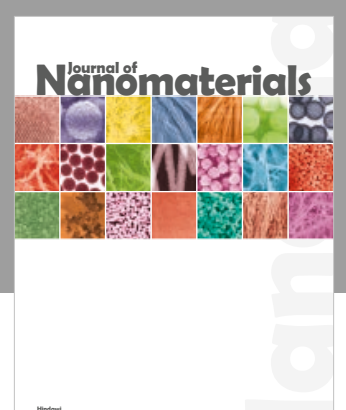

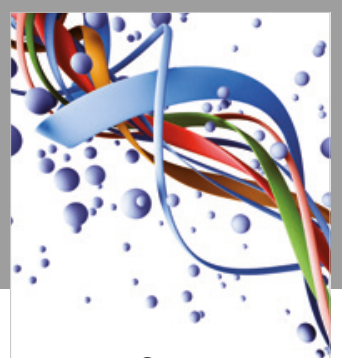

Scientifica

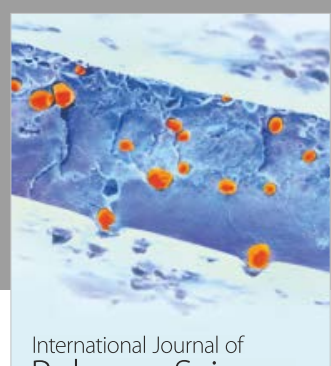

Polymer Science

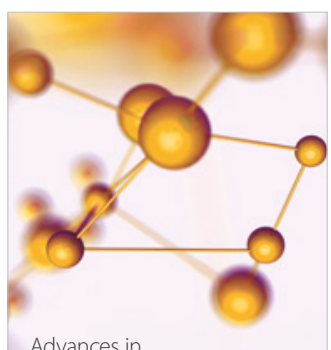

Physical Chemistry
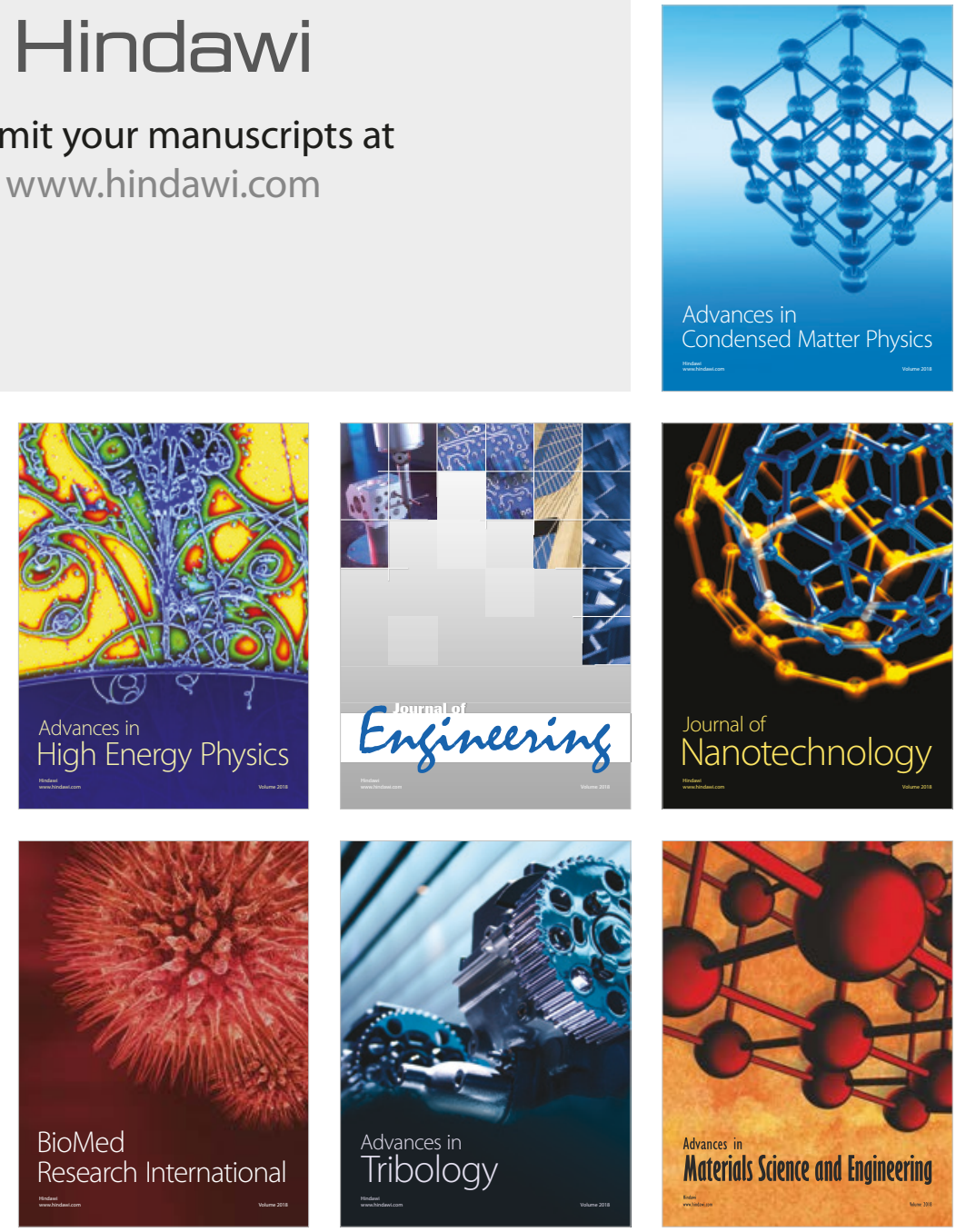